\title{
Melanocitoma associado à membrana neovascular sub-retiniana
}

\section{Melanocytoma associated to subretinal neovascular membrane}

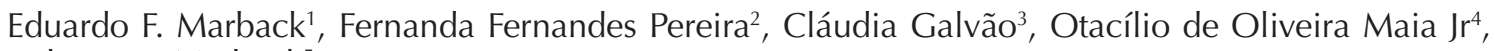
Roberto L. Marback ${ }^{5}$

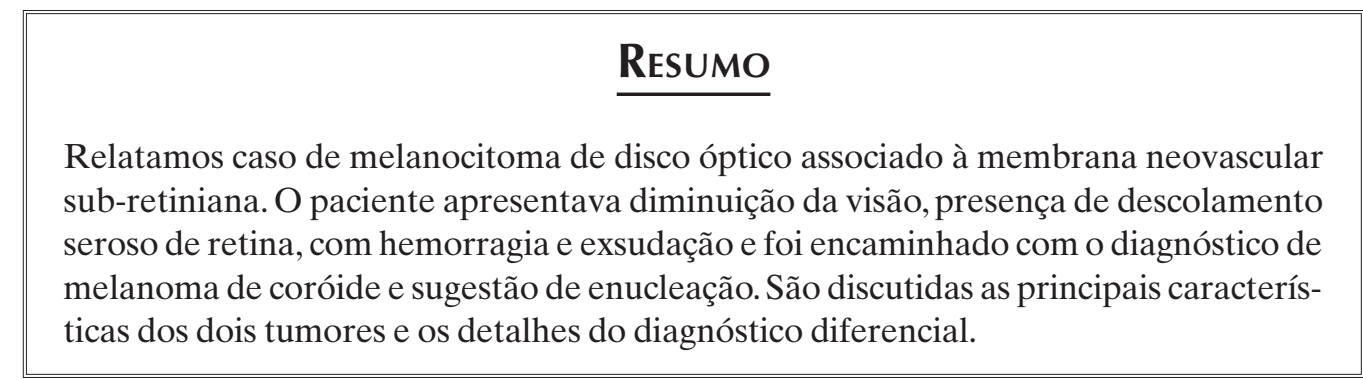

Descritores: Neoplasias do nervo óptico; Neoplasias do nervo óptico/complicações;

Melanoma/diagnóstico; Neovascularização coroidal

\footnotetext{
'Doutor, Médico Oftalmologista do Hospital Universitário Professor Edgard Santos da Universidade Federal da Bahia; Médico Oftalmologista do Hospital São Rafael, Fundação Monte Tabor - Salvador BA, Brasil;

${ }^{2}$ Aluna do Curso de Especialização em Oftalmologia da Universidade Federal da Bahia - UFBA - Salvador - BA, Brasil; ${ }^{3}$ Médica do Hospital Universitário Professor Edgard Santos da Universidade Federal da Bahia - UFBA - Salvador- BA, Brasil;

${ }^{4}$ Doutor, Professor Substituto de Oftalmologia da Universidade Federal da Bahia - UFBA - Salvador - BA, Brasil; Médico Oftalmologista do Hospital São Rafael, Fundação Monte Tabor - Salvador (BA), Brasil;

${ }^{5}$ Professor Titular de Oftalmologia da Universidade Federal da Bahia - UFBA - Salvador - BA, Brasil; Chefe do Serviço de Oftalmologia do Hospital São Rafael, Fundação Monte Tabor - Salvador (BA), Brasil.

Trabalho realizado no Hospital Universitário Prof. Edgard Santos da Universidade Federal da Bahia - UFBA - Salvador - BA, Brasil

Recebido para publicação em: 8/6/2009 - Aceito para publicação em 10/9/2009
} 


\section{INTRODUÇÃO}

$\mathbf{M}$ elanocitoma do disco óptico é um tumor benigno raro, usualmente assintomático e estacionário. Habitualmente seu diagnóstico é feito durante exame de rotina ou por queixas não relacionadas ao tumor, sendo mais comum em indivíduos de raças pigmentadas ${ }^{(1-4)}$

Quando ocorre diminuição de visão é usualmente causada por necrose espontânea do tumor, levando a exsudação e neuroretinite, compressão do nervo óptico e oclusão venosa ${ }^{(1,3,4)}$. A transformação maligna de um melanocitoma, embora possível, é extremamente rara ocorrendo entre 1 a $2 \%$ dos $\operatorname{casos}^{(2-4)}$.

Relatamos um caso de melanocitoma do disco óptico em paciente com membrana neovascular subretiniana encaminhado como melanoma uveal.

\section{Relato do caso}

Paciente do sexo masculino, pardo, 40 anos, com queixa de diminuição da visão progressiva em olho direito $(\mathrm{OD})$ há nove meses. Foi encaminhado ao nosso serviço com suspeita de melanoma de coróide e sugestão de enucleação do olho afetado.

Ao exame apresentava melhor acuidade visual corrigida de 0,1 em OD e 1,0 em olho esquerdo (OE). À oftalmoscopia observava-se tumor densamente pigmentado em metade superior do nervo óptico com bordas de aspecto aveludado. Exibia descolamento plano de retina em polo posterior com exsudação e hemorragia sub-retinianas, área espessada e de coloração amarelada em região macular, além de intenso pregueamento da membrana limitante interna (Figura 1A).

A ecografia do OD evidenciou lesão sobrelevada em área papilar, com extensão temporal, além de aparente elevação focal da retinia perilesional. O tumor media $2,4 \mathrm{~mm}$ de altura e 11,3 X 6,6mm de base. A angiofluoresceinografia (AFG) revelou hipofluorescência por bloqueio na topografia da lesão de nervo óptico e presença de área de hiperfluorescência precoce com limites bem definidos na região adjacente ao tumor no feixe papilo-macular. Na fase tardia do exame foi observado borramento dos limites da lesão, aspecto compatível com membrana neovascular do tipo clássica, adjacente ao tumor (Figura 1B-E).

O diagnóstico clínico foi de membrana neovascular sub-retiniana associada à melanocitoma. Devido ao estado avançado da membrana neovascular, já com aspecto cicatricial, optou-se pelo acompanhamento da mesma. Após um mês, paciente retornou exibindo

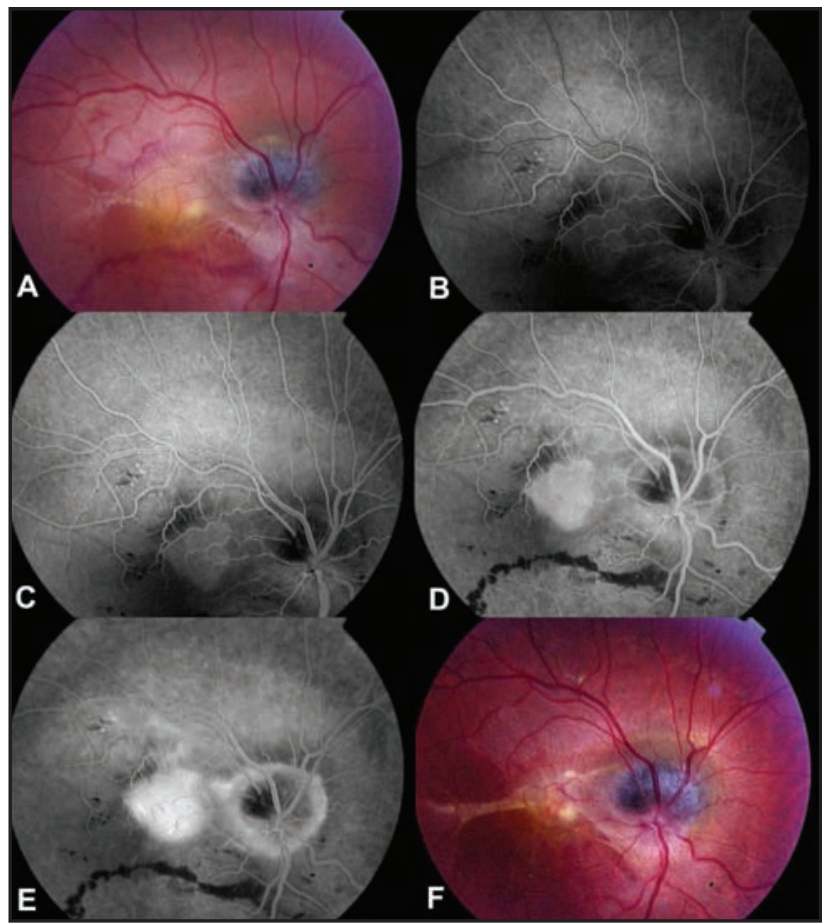

Figura 1A: Retinografia inicial exibindo tumor pigmentado na metade superior do disco óptico, elevação retiniana, lesão amarelada submacular e hemorragia sub-retiniana inferior; B-E: Angiofluoresceinografia; Lesão macular hiperfluorescente em fase precoce, com borramento tardio e tumor hipofluorescente sobre o disco óptico; F: Retinografia após um mês mostrando absorção do fluido sub-retiniano e lesão macular de aspecto cicatricial

absorção do fluído sub-retiniano com lesão pigmentada sobre o disco óptico estável (Figura 1F), sendo programado acompanhamento fotográfico semestral.

\section{DısCUSSÃo}

Apesar da natureza benigna do melanocitoma de disco óptico, pacientes com este tumor frequentemente são encaminhados para afastar a possibilidade de melanoma. No passado, muitos destes pacientes foram submetidos a enucleação com diagnóstico de melanoma de disco óptico ${ }^{(1)}$. A elucidação da natureza benigna do tumor se deve aos estudos histopatológicos destes olhos e ao acompanhamento de pacientes que se recusaram a fazer enucleação, comprovando que muitos destes tumores permaneciam estáveis ao longo dos anos ${ }^{(1)}$.

O melanocitoma do nervo óptico pode causar diminuição da visão em até $25 \%$ dos pacientes ${ }^{(1-4)}$. Tipicamente a baixa de visão é causada por exsudação subretiniana e necrose tumoral, levando a neuroretinite ou oclusões vasculares ${ }^{(2-4)}$. Crescimento tumoral documentado pode ser visto em aproximadamente $15 \%$ dos ca- 
$\operatorname{sos}^{(1-4)}$. A transformação maligna é bastante rara, com ocorrência estimada entre 1 a $2 \%$ dos $\operatorname{casos}^{(1-4)}$.

No caso descrito, a diminuição da acuidade visual, o descolamento seroso de retina e hemorragia, e exsudação sub-retinianas em um olho que exibia tumor pigmentado sobre o disco óptico motivou o encaminhamento com o suposto diagnóstico de melanoma. A AFG, embora não seja exame fundamental para o diagnóstico de melanoma uveal, em casos difíceis como este, pode se mostrar de grande valia ao evidenciar lesão pigmentada sobre o disco, com total bloqueio da fluorescência associada à membrana neovascular subretiniana. Assim, foi possível especular que a exsudação e a baixa de visão estavam sendo causadas pela membrana e não pelo crescimento do tumor pigmentado de disco óptico. O aparecimento de membrana neovascular sub-retiniana é uma complicação possível em qualquer do polo posterior sendo relacionada a ruptura na membrana de Bruch e possível produção de fatores angiogênicos pelo tumor. Na série de 115 melanocitomas descrita por Shields e colaboradores, neovascularização sub-retiniana foi encontrada em apenas um paciente ${ }^{(3)}$. Em dois outros relatos, o quadro clínico foi muito similar ao do nosso paciente, com iní-

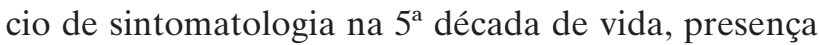
de exsudatos e hemorragia sub-retiniana ${ }^{(5,6)}$. Em um dos relatos o paciente foi submetido à remoção cirúrgica da membrana neovascular com bom resultado funcional final ${ }^{(6)}$. Chalam e colaboradores descrevem um caso tratado de maneira bem-sucedida com terapia fotodinâmica ${ }^{(7)}$. Não encontramos referência ao uso de injeção intravítrea de antiangiogênicos para tratamento da membrana associada ao melanocitoma. Em nosso paciente, devido ao estado avançado da membrana com aparente gliose retiniana, optamos apenas pelo acompanhamento.

Chama a atenção o encaminhamento com suspeita de melanoma uveal e sugestão de enucleação. A diferenciação entre um melanocitoma causando baixa de visão e um pequeno melanoma peripapilar com invasão de nervo óptico pode ser difícil. A invasão do nervo óptico por melanoma uveal é evento raro, com ocorrência estimada em apenas 0,8 a 3,7\% dos casos, podendo acontecer tanto em tumores negligenciados, que preenchem toda a cavidade vítrea, como em pequenos melanomas peripapilares ${ }^{(8-}$ 11). Para dificultar ainda mais, sempre devemos lembrar que a transformação maligna em um melanocitoma embora rara, pode ocorrer existindo até mesmo a descrição de tumores com uma porção benigna e outra maligna, o que pode inclusive resultar em interpretação errônea em biópsia de aspiração por agulha fina ${ }^{(2,3,12)}$.

Apesar das dificuldades no diagnóstico diferencial entre melanocitoma com baixa de visão e pequeno melanoma com invasão de nervo óptico, alguns dados podem ser úteis. $\mathrm{O}$ aspecto oftalmoscópico do melanocitoma é de tumor densamente pigmentado, com aspecto negro ou castanho, superfície aveludada, com frequente componente retiniano ${ }^{(1-4)}$. Já o melanoma usualmente exibe pigmentação menos densa e menos homogênea, com predominância do componente coroidal $^{(10)}$. A AFG no melanocitoma mostra intensa hipofluorescência por bloqueio devido à densa pigmentação do tumor, enquanto o melanoma costuma exibir vascularização intrínseca ${ }^{(3,4,10)}$. A ecografia, de grande valia no diagnóstico diferencial dos tumores oculares, tem papel limitado nesta situação devido às pequenas dimensões das lesões. Um pequeno melanoma com invasão de nervo óptico poderia apresentar aspecto oftalmoscópico (exsudação e descolamento de retina) e sintomatologia (baixa visual), muito similares aos do caso em questão ${ }^{(10)}$. Os achados que mais contribuíram para a elucidação diagnóstica em nosso caso foram ausência de dupla circulação à AFG e presença de membrana neovascular justificando a baixa de visão. Frente a tal dúvida diagnóstica, uma alternativa é observar a evolução do tumor em um período relativamente curto, como fizemos. Caso se tratasse de melanoma, poderíamos esperar uma evolução do quadro com aumento do tumor e piora da acuidade visual, orientando de forma correta o tratamento.

\section{Abstract}

We report a case of optic disc melonocytoma associated to subretinal neovascular membrane. The patient presented with low visual acuity, serous retinal detachment with haemorrhage and exudates and was referred with the diagnosis of melanoma and an orientation to enucleate the eye. We discuss the major aspects of both tumors and the details of differential diagnostic.

Keywords: Optic nerve neoplasms; Optic nerve neoplasms/complications; Melanoma/diagnosis; Choroidal neovascularization

\section{ReFERÊNCIAS}

1. Zimmerman LE, Garron LK. Melanocytoma of the optic disk. Int Ophthalmol Clin. 1962; 2:431-4 .

2. Joffe L, Shields JA, Osher RH, Gass JD. Clinical and followup studies of melanocytomas of the optic disc. Ophthalmology.1979; 86(6):1067-83. 
3. Shields JA, Demirci H, Mashayekhi A, Shields CL.Melanocytoma of optic disc in 115 cases: the 2004 Samuel Johnson Memorial Lecture, part 1. Ophthalmology. 2004; 111(9):1739-46.

4. Shields JA, Demirci H, Mashayekhi A, Eagle RC Jr, Shields CL. Melanocytoma of optic disc: A review. Surv Ophthalmol. 2006;51(2):93-104. Review.

5. Chalam KV, Shah GY, Shah VA, Rappaport KD. Choroidal neovascular membrane associated with melanocytoma of the optic nerve. Retina. 2006;26(6):703-4.

6. Tran VH, Bovey EH, Uffer S, Zografos L. Peripapillary choroidal neovascularization associated with melanocytoma of the optic disc: a clinicopathologic case report. Graefe's Arch Clin Exp Ophthalmol. 2006; 244(10): 1367-9.

7. Chalam KV, Gupta SK, Shah GY, Agarwal S. Successful management of melanocytoma-associated choroidal neovascularization with photodynamic therapy. Eur J Ophthalmol. 2006;16(5):776-8.

8. Coupland SE, Campbell I, Damato B. Routes of extraocular extension of uveal melanoma: risk factors and influence on survival probability. Ophthalmology. 2008;115(10):1778-85.
9. Christmas NJ, Mead MD, Richardson EP, Albert DM. Secondary optic nerve tumors. Surv Ophthalmol. 1991; 36(3):196206.

10. Marback EF, Arias VEA, Gonzaga RL, Pinto CA, Erwenne $\mathrm{CM}$. Invasão do nervo óptico por pequeno melanoma maligno da coróide. Arq Bras Oftalmol. 2003; 66(3):369-72.

11. Marback EF, Fernades PM, Castelo Branco B, Marback RL, Sento Sé DC. Invasão do nervo óptico por melanoma malígno da úvea. Arq Bras Oftalmol. 1996;59(6):611-13.

12. Shetlar DJ, Folberg R, Gass JDM. Choroidal malignant melanoma associated with a melanocytoma. Retina. 1999;19(4):346-9.

\section{Endereço para Correspondência:}

Eduardo F Marback

Rua Eduardo José dos Santos, 147, sala 808, Ondina

Salvador (BA), Brasil.

CEP 41940-455

Tel 71 3235-9368 